\title{
Genome sequence of the homoacetogenic bacterium Holophaga foetida type strain (TMBS4 ${ }^{\mathrm{T}}$ )
}

\author{
lain Anderson ${ }^{1}$, Brittany Held ${ }^{1,2}$, Alla Lapidus ${ }^{1}$, Matt Nolan ${ }^{1}$, Susan Lucas ${ }^{1}$, Hope Tice ${ }^{1}$, \\ Tijana Glavina Del Rio ${ }^{1}$, Jan-Fang Cheng ${ }^{1}$, Cliff Han ${ }^{1,2}$, Roxanne Tapia ${ }^{1,2}$, Lynne A. Good- \\ win $^{1,2}$, Sam Pitluck ${ }^{1}$, Konstantinos Liolios ${ }^{1}$, Konstantinos Mavromatis ${ }^{1}$, Ioanna Pagani ${ }^{1}$, \\ Natalia Ivanova ${ }^{1}$, Natalia Mikhailova ${ }^{1}$, Amrita Pati ${ }^{1}$, Amy Chen ${ }^{3}$, Krishna Palaniappan ${ }^{3}$, Mir- \\ iam Land ${ }^{1,4}$, Evelyne-Marie Brambilla ${ }^{6}$, Manfred Rohde ${ }^{5}$, Stefan Spring ${ }^{6}$, Markus Göker ${ }^{6}$, \\ John C. Detter ${ }^{1,2}$, Tanja Woyke ${ }^{1}$, James Bristow ${ }^{1}$, Jonathan A. Eisen ${ }^{1,7}$, Victor Markowitz ${ }^{3}$, \\ Philip Hugenholtz ${ }^{1,8}$, Hans-Peter Klenk ${ }^{6 *}$, Nikos C. Kyrpides ${ }^{1 *}$ \\ ${ }^{1}$ DOE Joint Genome Institute, Walnut Creek, California, USA \\ ${ }^{2}$ Los Alamos National Laboratory, Bioscience Division, Los Alamos, New Mexico, USA \\ ${ }^{3}$ Biological Data Management and Technology Center, Lawrence Berkeley National \\ Laboratory, Berkeley, California, USA \\ ${ }^{4}$ Oak Ridge National Laboratory, Oak Ridge, Tennessee, USA \\ ${ }^{5}$ HZI - Helmholtz Centre for Infection Research, Braunschweig, Germany \\ ${ }^{6}$ Leibniz Institute DSMZ - German Collection of Microorganisms and Cell Cultures, \\ Braunschweig, Germany \\ ${ }^{7}$ University of California Davis Genome Center, Davis, California, USA \\ ${ }^{8}$ Australian Centre for Ecogenomics, School of Chemistry and Molecular Biosciences, The \\ University of Queensland, Brisbane, Australia
}

*Corresponding authors: Nikos C. Kyrpides (nckyrpides@lbl.gov) and Hans-Peter Klenk (hpk@dsmz.de)

Keywords: anaerobic, non-motile, Gram-negative, mesophilic, chemoorganotrophic, sulfidemethylation, fresh water mud, Acidobacteria, Holophagaceae, GEBA

\footnotetext{
Holophaga foetida Liesack et al. 1995 is a member of the phylum Acidobacteria and is of interest for its ability to anaerobically degrade aromatic compounds and for its production of volatile sulfur compounds through a unique pathway. The genome of $\mathrm{H}$. foetida strain $\mathrm{TMBS}^{\mathrm{T}}$ is the first to be sequenced for a representative of the class Holophagae. Here we describe the features of this organism, together with the complete genome sequence (improved high quality draft), and annotation. The 4,127,237 bp long chromosome with its 3,615 protein-coding and 57 RNA genes is a part of the Genomic Encyclopedia of Bacteria and Archaea project.
}

\section{Introduction}

Strain TMBS4T (= DSM 6591) is the type strain of the species Holophaga foetida [1], which is the type species of the monospecific genus Holophaga [1,2]. The genus Holophaga is the type genus of the family Holophagaceae [3] in the order Holophagales [3] within the class Holophagae [3]. The genus name was derived from a combination of the Neo-Greek term holos, whole, and the Greek term phagein, to eat, meaning eating it all [1]; the species epithet was derived from the Latin adjective foetidus, smelling, stinking, referring to the production of foul-smelling methanethiol and dimethylsulfide [1]. Strain TMBS4T was originally isolated from a black anoxic freshwater mud sample from a ditch near Konstanz, Germany [4]. It was found to transfer methyl groups from methoxylated aromatic compounds to sulfide, forming methanethiol and dimethylsulfide [4]. Dimethylsulfide plays an important role in atmospheric chemistry, and is produced mainly by marine bacteria from dimethylsulfoniopropionate (reviewed in [5]). 
Anderson et al.

The production of dimethylsulfide from methoxylated aromatic compounds represents a unique pathway for production of this important compound. Strain TMBS4T anaerobically degrades several aromatic compounds to acetate $[1,4]$. The only other cultured species within the order Holophagales is Geothrix fermentans, which is also an anaerobe but degrades small organic acids and fatty acids using Fe(III) as an electron acceptor [6]. Here we present a summary classification and a set of features for $H$. foetida TMBS4T, together with the description of the genomic sequencing and annotation.

\section{Classification and features}

A representative genomic $16 \mathrm{~S}$ rRNA sequence of H. foetida TMBS4T was compared using NCBI BLAST $[7,8]$ under default settings (e.g., considering only the high-scoring segment pairs (HSPs) from the best 250 hits) with the most recent release of the Greengenes database [9] and the relative frequencies of taxa and keywords (reduced to their stem [10]) were determined, weighted by BLAST scores. The most frequently occurring genera were Holophaga (52.9\%), Geothrix (33.7\%) and Acidobacterium (13.4\%) (5 hits in total). Regarding the two hits to sequences from members of the species, the average identity within HSPs was $99.7 \%$, whereas the average coverage by HSPs was $100.0 \%$. Among all other species, the one yielding the highest score was $G$. fermentans (NR_036779), which corresponded to an identity of $91.6 \%$ and an HSP coverage of $97.8 \%$. (Note that the Greengenes database uses the INSDC (= EMBL/NCBI/DDBJ) annotation, which is not an authoritative source for nomenclature or classification.) The highest-scoring environmental sequence was DQ676369 ('Archaeal sediment and plankton freshwater pond suboxic freshwaterpond clone MVP-105'), which showed an identity of $97.6 \%$ and an HSP coverage of $94.9 \%$. The most frequently occurring keywords within the labels of all environmental samples which yielded hits were 'lake' (6.2\%), 'aquat' (4.6\%), 'gatun, rank' (4.3\%), 'soil' (3.4\%) and 'microbi' (2.1\%) (245 hits in total). The most frequently occurring keywords within the labels of those environmental samples which yielded hits of a higher score than the highest scoring species were 'situ' (3.3\%), 'microbi' (3.0\%), 'groundwat' (2.8\%), 'activ'
(2.5\%) and 'aquif' (2.5\%) (42 hits in total), all of which are keywords with biological meaning fitting the environment from which strain TMBS4 was isolated.

Figure 1 shows the phylogenetic neighborhood of $H$. foetida in a $16 \mathrm{~S}$ rRNA based tree. The sequences of the two identical 16S rRNA gene copies in the genome differ by two nucleotides from the previously published 16S rRNA sequence (X77215), which contains one ambiguous base call.

H. foetida TMBS4T is Gram-negative, and an electron micrograph shows an inner and outer membrane [1]. Cells are rod-shaped, 1-3 $\mu \mathrm{m}$ long and 0.5-0.7 $\mu \mathrm{m}$ wide [1,4] (Figure 2). No motility was observed [1,4], although the genome is rich in genes classified under 'cell motility' (152 genes). Growth was observed between $10^{\circ} \mathrm{C}$ and $35^{\circ} \mathrm{C}$ with an optimum at $28-32^{\circ} \mathrm{C}[1,4]$. The $\mathrm{pH}$ range for growth was 5.5-8.0 with 6.8-7.5 as the optimum $[1,4]$. The salinity range for growth was $1-15 \mathrm{~g} / \mathrm{l}$ $\mathrm{NaCl}$ [4]. Aromatic compounds utilized by TMBS4 ${ }^{\mathrm{T}}$ include 3,4,5-trimethoxybenzoate, syringate, 5hydroxyvanillate, phloroglucinol monomethyl ether, sinapate, ferulate, caffeate, gallate, 2,4,6trihydroxybenzoate, pyrogallol, and phloroglucinol $[1,4]$. The fastest growth occurred with syringate [4]. When sulfide was present in the medium, methyl groups from aromatic compounds were used to form methanethiol and dimethylsulfide $[1,4]$. Strain TMBS4 ${ }^{\mathrm{T}}$ could also grow with $\mathrm{CO}$ or $\mathrm{CO}_{2}$ as methyl acceptors, and acetyl-CoA synthase activity was detected [26]. Growth was also observed on pyruvate $[1,4]$.

\section{Genome sequencing and annotation Genome project history}

This organism was selected for sequencing on the basis of its phylogenetic position [27], and is part of the Genomic Encyclopedia of Bacteria and Archaea project [28]. The genome project is deposited in the Genomes On Line Database [17] and the complete genome sequence is deposited in GenBank. Sequencing, finishing and annotation were performed by the DOE Joint Genome Institute (JGI). A summary of the project information is shown in Table 2. 


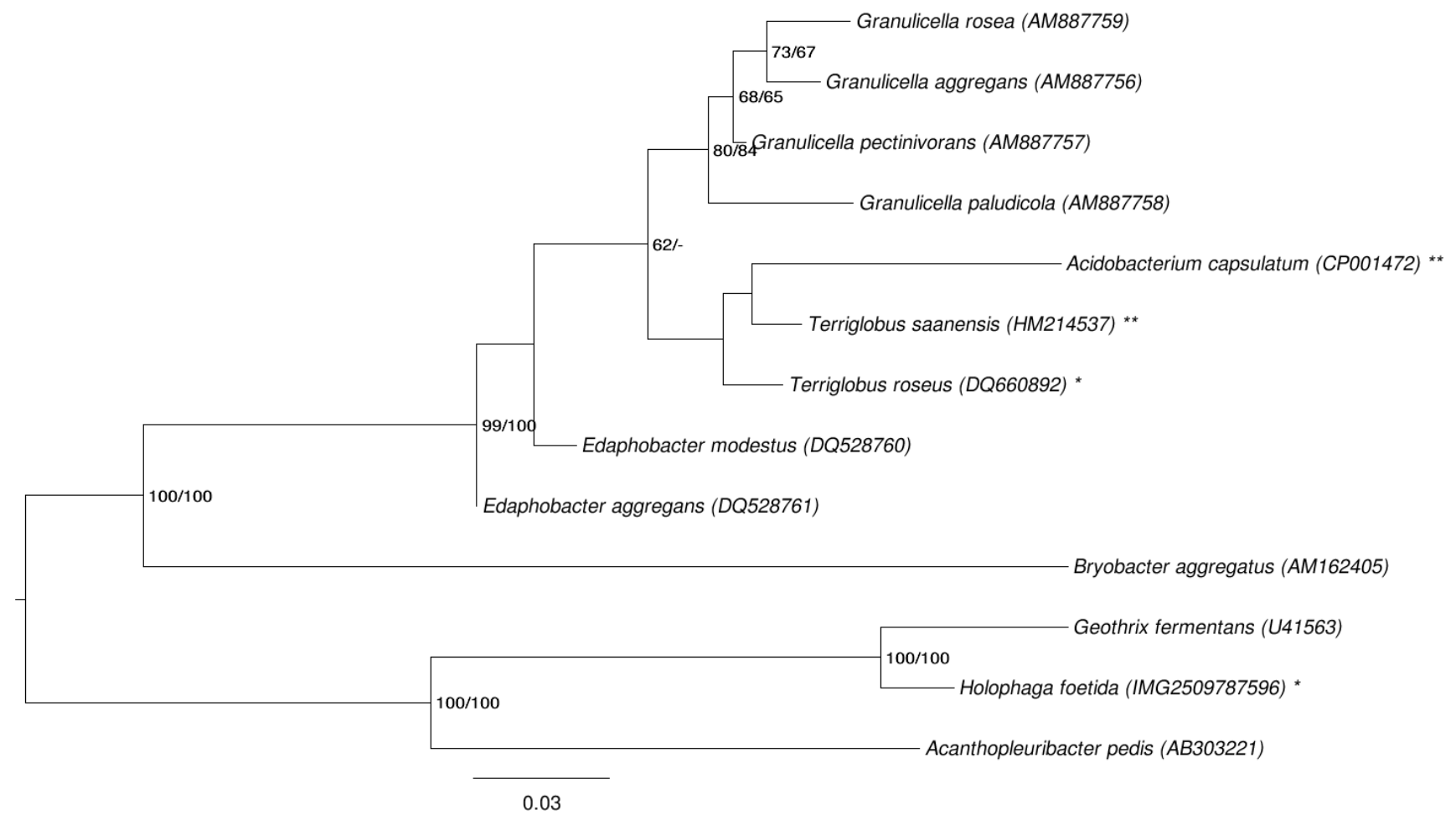

Figure 1. Phylogenetic tree highlighting the position of $H$. foetida relative to the type strains of the other species within the phylum 'Acidobacteria'. The tree was inferred from 1,395 aligned characters [11,12] of the $16 \mathrm{~S}$ rRNA gene sequence under the maximum likelihood $(\mathrm{ML})$ criterion [13]. Rooting was done initially using the midpoint method [14] and then checked for its agreement with the current classification (Table 1). The branches are scaled in terms of the expected number of substitutions per site. Numbers adjacent to the branches are support values from $400 \mathrm{ML}$ bootstrap replicates [15] (left) and from 1,000 maximum-parsimony bootstrap replicates [16] (right) if larger than $60 \%$. Lineages with type strain genome sequencing projects registered in GOLD [17] are labeled with one asterisk, those also listed as 'Complete and Published' with two asterisks [18] (see CP002467 for Terriglobus saanensis).

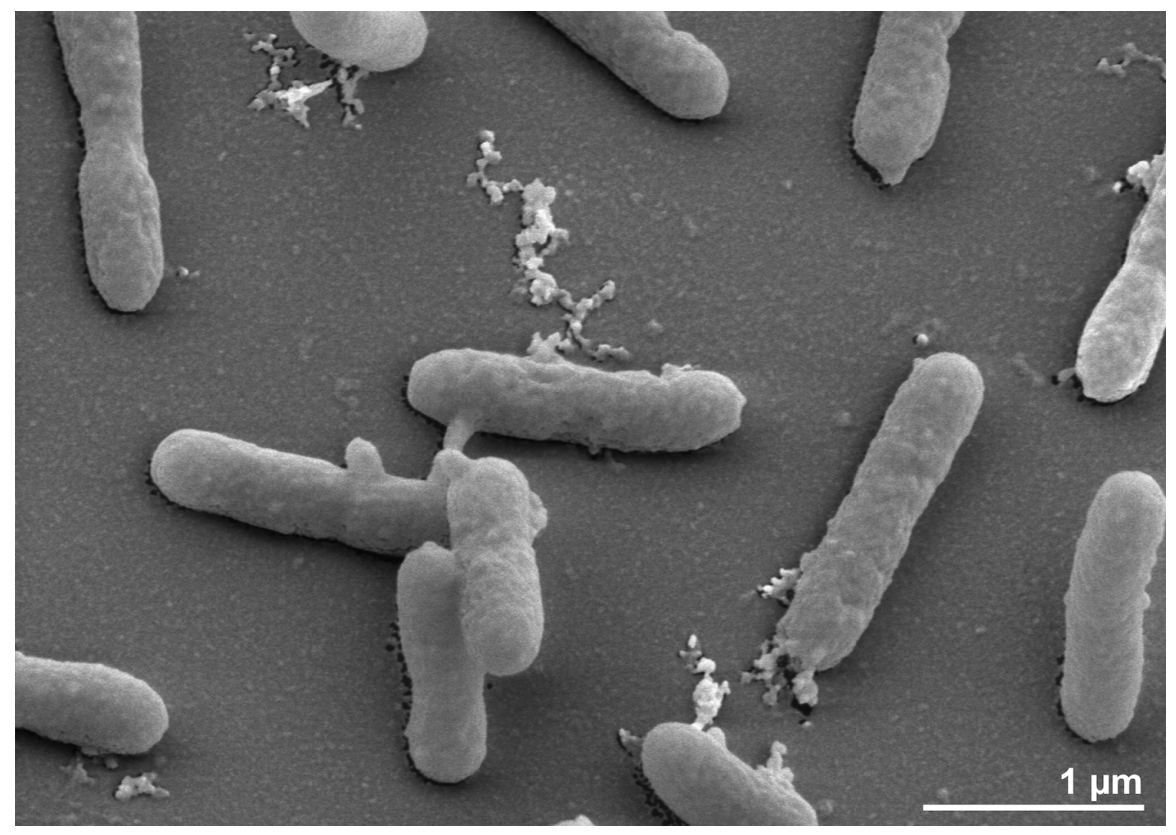

Figure 2. Scanning electron micrograph of $H$. foetida TMBS4 ${ }^{\top}$ 
Anderson et al.

Table 1. Classification and general features of H. foetida TMBS4 ${ }^{\top}$ according to the MIGS recommendations [19].

\begin{tabular}{|c|c|c|c|}
\hline MIGS ID & Property & Term & Evidence code \\
\hline & \multirow{8}{*}{ Current classification } & Domain Bacteria & TAS [20] \\
\hline & & Phylum Acidobacteria & TAS $[21,22]$ \\
\hline & & Class Holophagae & TAS [3] \\
\hline & & Order Holophagales & TAS [3] \\
\hline & & Family Holophagaceae & TAS [3] \\
\hline & & Genus Holophaga & TAS $[1,23]$ \\
\hline & & Species Holophaga foetida & TAS $[1,23]$ \\
\hline & & Type-strain TMBS4 & TAS [1] \\
\hline & Gram stain & negative & TAS [1] \\
\hline & Cell shape & rod-shaped & TAS $[1,4]$ \\
\hline & Motility & non-motile & TAS $[1,4]$ \\
\hline & Sporulation & non-sporulating & TAS [4] \\
\hline & Temperature range & mesophile, $10-35^{\circ} \mathrm{C}$ & TAS $[1,4]$ \\
\hline & Optimum temperature & $28-32^{\circ} \mathrm{C}$ & TAS $[1,4]$ \\
\hline & Salinity & $1-15 \mathrm{~g} / \mathrm{l} \mathrm{NaCl}$ & TAS [4] \\
\hline \multirow[t]{3}{*}{ MIGS-22 } & Oxygen requirement & obligate anaerobe & TAS $[1,4]$ \\
\hline & Carbon source & $\begin{array}{l}\text { methoxylated and hydroxylated aromatic compounds, } \\
\text { pyruvate }\end{array}$ & TAS [1] \\
\hline & Energy metabolism & chemoorganotroph & TAS [1] \\
\hline MIGS-6 & Habitat & freshwater mud & TAS [1] \\
\hline MIGS-15 & Biotic relationship & free living & TAS [1] \\
\hline \multirow[t]{2}{*}{ MIGS-14 } & Pathogenicity & none & NAS \\
\hline & Biosafety level & 1 & TAS [24] \\
\hline MIGS-23.1 & Isolation & freshwater mud & TAS [1] \\
\hline MIGS-4 & Geographic location & near Konstanz, Germany & TAS [1] \\
\hline MIGS-5 & Sample collection time & 1989 or earlier & NAS \\
\hline MIGS-4.1 & Latitude & 47.663 & TAS [1] \\
\hline MIGS-4.2 & Longitude & 9.175 & TAS [1] \\
\hline MIGS-4.3 & Depth & not reported & \\
\hline MIGS-4.4 & Altitude & not reported & \\
\hline
\end{tabular}

Evidence codes - TAS: Traceable Author Statement (i.e., a direct report exists in the literature); NAS: Non-traceable Author Statement (i.e., not directly observed for the living, isolated sample, but based on a generally accepted property for the species, or anecdotal evidence). These evidence codes are from the Gene Ontology project [25]. 


\section{Growth conditions and DNA isolation}

H. foetida strain TMBS4T, DSM 6591, was grown anaerobically in DSMZ medium 559 (TMBS4 medium) [29] at $30^{\circ} \mathrm{C}$. DNA was isolated from $0.5-1 \mathrm{~g}$ of cell paste using MasterPure Gram-positive DNA purification kit (Epicentre MGP04100) following the standard protocol as recommended by the manufacturer with modification st/DL for cell lysis as described in Wu et al. 2009 [28]. DNA is available through the DNA Bank Network [30].

\section{Genome sequencing and assembly}

The genome was sequenced using a combination of Illumina and 454 sequencing platforms. All general aspects of library construction and sequencing can be found at the JGI website [31]. Pyrosequencing reads were assembled using the Newbler assembler (Roche). The initial Newbler assembly consisting of 186 contigs in two scaffolds was converted into a phrap [32] assembly by making fake reads from the consensus, to collect the read pairs in the 454 paired end library. Illumina GAii sequencing data $(9,124.2 \mathrm{Mb})$ was assembled with Velvet [33] and the consensus sequences were shredded into $1.5 \mathrm{~kb}$ overlapped fake reads and assembled together with the 454 data. The 454 draft assembly was based on 135.9 $\mathrm{Mb}$ of 454 draft data and all of the 454 paired end data. Newbler parameters are -consed -a 50 -l 350 -g -m -ml 20. The Phred/Phrap/Consed software package [32] was used for sequence assembly and quality assessment in the subsequent finishing process. After the shotgun stage, reads were assembled with parallel phrap (High Performance Software, LLC). Possible mis-assemblies were corrected with gapResolution (C. Han, unpublished), Dupfinisher [34], or sequencing cloned bridging PCR fragments with subcloning. Gaps between contigs were closed by editing in Consed, by PCR and by Bubble PCR primer walks (J.-F. Chang, unpublished). A total of 192 additional reactions were necessary to close some gaps and to raise the quality of the assembled sequence. Illumina reads were also used to correct potential base errors and increase consensus quality using a software Polisher developed at JGI [35]. The error rate of the assembled genome sequence is less than 1 in 100,000 . Together, the combination of the Illumina and 454 sequencing platforms provided $2,192.4 \times$ coverage of the genome. The final assembly contained 461,984 pyrosequence and $120,055,671$ Illumina reads and consists of 39 contigs organized in three scaffolds.

Table 2. Genome sequencing project information

\begin{tabular}{|c|c|c|}
\hline MIGS ID & Property & Term \\
\hline MIGS-31 & Finishing quality & Improved high quality draft \\
\hline MIGS-28 & Libraries used & $\begin{array}{l}\text { Four genomic libraries: two } 454 \text { pyrosequence standard libraries, one } \\
454 \text { PE library (11.5 kb insert size), one Illumina library }\end{array}$ \\
\hline MIGS-29 & Sequencing platforms & Illumina GAii, 454 GS FLX Titanium \\
\hline MIGS-31.2 & Sequencing coverage & $2,172.4 \times$ Illumina; $20.0 \times$ pyrosequence \\
\hline MIGS-30 & Assemblers & Newbler version 2.3, Velvet version 1.0.13, phrap version SPS - 4.24 \\
\hline \multirow[t]{6}{*}{ MIGS-32 } & Gene calling method & Prodigal \\
\hline & INSDC ID & AGSB00000000 \\
\hline & GenBank Date of Release & January 12, 2012 \\
\hline & GOLD ID & Gi05348 \\
\hline & NCBI project ID & 53485 \\
\hline & Database: IMG-GEBA & 2509601028 \\
\hline \multirow[t]{2}{*}{ MIGS-13 } & Source material identifier & DSM 6591 \\
\hline & Project relevance & Tree of Life, GEBA, Bioremediation \\
\hline
\end{tabular}




\section{Genome annotation}

Genes were identified using Prodigal [36] as part of the Oak Ridge National Laboratory genome annotation pipeline, followed by a round of manual curation using the JGI GenePRIMP pipeline [37]. The predicted CDSs were translated and used to search the National Center for Biotechnology Information (NCBI) non-redundant database, UniProt, TIGRFam, Pfam, PRIAM, KEGG, COG, and InterPro databases. These data sources were combined to assert a product description for each predicted protein. Noncoding genes and miscellaneous features were predicted using tRNAscan-SE [38], RNAMMer [39], Rfam [40], TMHMM [41], and signalP [42].

\section{Genome properties}

The genome in its current assembly consists of three scaffolds with lengths of $3,443,192 \mathrm{bp}$, $677,300 \mathrm{bp}$ and $6,745 \mathrm{bp}$ and a $63.0 \% \mathrm{G}+\mathrm{C}$ content (Table 3 and Figure 3). Of the 3,672 predicted genes, 3,615 were protein-coding genes, and 57 RNAs; 76 pseudogenes were also identified. The majority of the protein-coding genes (74.3\%) were assigned a putative function while the remaining ones were annotated as hypothetical proteins. The distribution of genes into COGs functional categories is presented in Table 4.

Table 3. Genome Statistics

\begin{tabular}{lrr}
\hline Attribute & Value & \% of Total \\
\hline Genome size (bp) & $4,127,237$ & $100.00 \%$ \\
DNA coding region (bp) & $3,689,184$ & $89.39 \%$ \\
DNA G+C content (bp) & $2,595,845$ & $62.95 \%$ \\
Number of scaffolds & 3 & \\
Total genes & 3,672 & $100.00 \%$ \\
RNA genes & 57 & $1.55 \%$ \\
rRNA operons & 2 & \\
tRNA genes & 47 & $1.28 \%$ \\
Protein-coding genes & 3,615 & $98.45 \%$ \\
Pseudo genes & 76 & $2.07 \%$ \\
Genes with function prediction (proteins) & 2,728 & $74.29 \%$ \\
Genes in paralog clusters & 1,916 & $52.18 \%$ \\
Genes assigned to COGs & 2,788 & $75.93 \%$ \\
Genes assigned Pfam domains & 2,729 & $74.32 \%$ \\
Genes with signal peptides & 746 & $20.32 \%$ \\
Genes with transmembrane helices & 703 & $19.14 \%$ \\
CRISPR repeats & 1 & \\
\hline
\end{tabular}




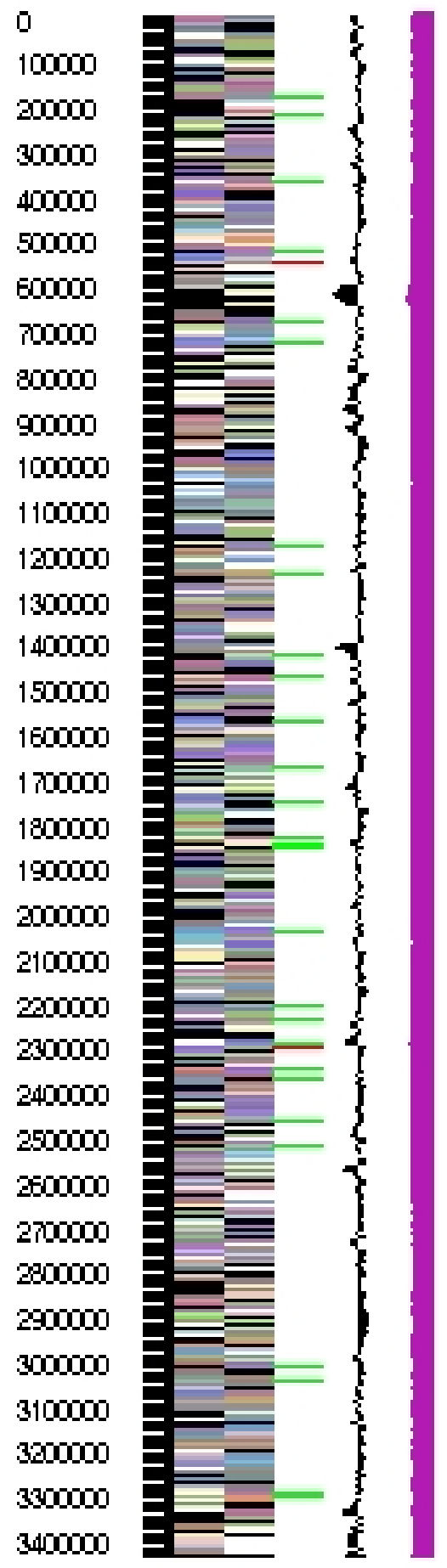

Figure 3. Graphical map of the largest scaffold. From left to the right: Genes on forward strand (color by COG categories), Genes on reverse strand (color by COG categories), RNA genes (tRNAs green, rRNAs red, other RNAs black), GC content, GC skew. 
Table 4. Number of genes associated with the general COG functional categories

\begin{tabular}{crrl}
\hline Code & value & \%age & Description \\
\hline J & 159 & 5.1 & Translation, ribosomal structure and biogenesis \\
A & 2 & 0.1 & RNA processing and modification \\
K & 254 & 8.1 & Transcription \\
L & 191 & 6.1 & Replication, recombination and repair \\
B & 0 & 0.0 & Chromatin structure and dynamics \\
D & 35 & 1.1 & Cell cycle control, cell division, chromosome partitioning \\
Y & 0 & 0.0 & Nuclear structure \\
V & 54 & 1.7 & Defense mechanisms \\
T & 267 & 8.5 & Signal transduction mechanisms \\
M & 194 & 6.2 & Cell wall/membrane biogenesis \\
N & 152 & 4.8 & Cell motility \\
Z & 1 & 0.0 & Cytoskeleton \\
W & 0 & 0.0 & Extracellular structures \\
U & 112 & 3.6 & Intracellular trafficking and secretion, and vesicular transport \\
O & 94 & 3.0 & Posttranslational modification, protein turnover, chaperones \\
C & 245 & 7.8 & Energy production and conversion \\
G & 120 & 3.8 & Carbohydrate transport and metabolism \\
E & 201 & 6.4 & Amino acid transport and metabolism \\
F & 69 & 2.2 & Nucleotide transport and metabolism \\
H & 183 & 5.8 & Coenzyme transport and metabolism \\
I & 87 & 2.8 & Lipid transport and metabolism \\
P & 135 & 4.3 & Inorganic ion transport and metabolism \\
Q & 32 & 1.0 & Secondary metabolites biosynthesis, transport and catabolism \\
R & 361 & 11.5 & General function prediction only \\
S & 201 & 6.4 & Function unknown \\
- & 884 & 24.1 & Not in COGs \\
\hline & & &
\end{tabular}

\section{Insights into the genome sequence}

$H$. foetida is known to utilize aromatic compounds through the phloroglucinol pathway, producing three molecules of acetate from the benzene ring. It is also capable of growing on methoxylated aromatic compounds, transferring methyl groups to sulfide or $\mathrm{CO}_{2}$ to produce dimethylsulfide or acetylCoA. Genes have not been identified for the enzymes of the phloroglucinol pathway with one exception: the transhydroxylase that converts pyrogallol to phloroglucinol, which has been identified in Pelobacter acidigallici [43]. This enzyme has two subunits, and genes with high similarity to these subunits are found in $H$. foetida. HolfoDRAFT_0037 and HolfoDRAFT_0041 have $74 \%$ and $88 \%$ similarity to the large subunit, while HolfoDRAFT_0036, HolfoDRAFT_0040, and HolfoDRAFT_0058 have 63\%, 73\%, and 65\% similarity to the small subunit.
H. foetida likely gains energy from the conversion of acetyl-CoA produced from aromatic compounds, pyruvate, and methyl groups from methoxylated aromatic compounds to acetate. Within the genome, there are two phosphotransacetylase genes (HolfoDRAFT_0402, HolfoDRAFT_1130) and two acetate kinase genes (HolfoDRAFT_1418, HolfoDRAFT_3547). Several candidates for pyruvate:ferredoxin oxidoreductase were found in the genome. This enzyme would produce acetylCoA that can be used to produce ATP and acetate. $H$. foetida can combine methyl groups with $\mathrm{CO}$ or $\mathrm{CO}_{2}$ to form acetyl-CoA, and acetyl-CoA synthase activity was detected [26]. Also within the genome, there are genes for a Moorella-type CO dehydrogenase/acetyl-CoA synthase (HolfoDRAFT_1152 and HolfoDRAFT_1153) and the two subunits of the corrinoid Fe-S protein (HolfoDRAFT_1154 and HolfoDRAFT_1157). 
H. foetida has potential symporters and $\mathrm{ABC}$ transporters for aromatic compounds. Four genes (HolfoDRAFT_0048, HolfoDRAFT_0224, HolfoDRAFT_0791, HolfoDRAFT_0858) belonging to the major facilitator superfamily have strong similarity to aromatic compound transporters of family 2.A.1.15. H. foetida has few $\mathrm{ABC}$ transporters for organic compounds, but it has 3 full transporters and 8 additional substrate binding proteins from family 4 . Some members of this family are amino acid transporters, but one member has been found to transport protocatechuate [44].

Systems for demethylation of methoxyaromatic compounds have been identified in Acetobacterium dehalogenans [45] and Moorella thermoacetica [46]. Methyl groups are transferred first to a corrinoid protein, then to tetrahydrofolate, by two methyltransferases. The genes for two sets of enzymes from $A$. dehalogenans have been sequenced [47]. The corrinoid proteins belong to COG5012,

\section{Acknowledgements}

We would like to gratefully acknowledge the help of Maren Schröder (DSMZ) for growing $\mathrm{H}$. foetida cultures. This work was performed under the auspices of the US Department of Energy's Office of Science, Biological and Environmental Research Program, and by the University of California, Lawrence Berkeley National Laboratory under contract No. DE-AC02-05CH11231, Lawrence

\section{References}

1. Liesack W, Bak F, Kreft JU, Stackebrandt E. Holophaga foetida gen. nov., sp. nov., a new, homoacetogenic bacterium degrading methoxylated aromatic compounds. Arch

Microbiol 1994; 162:85-90. PubMed

http://dx.doi.org/10.1007/BF00264378

2. Garrity G. NamesforLife. BrowserTool takes expertise out of the database and puts it right in the browser. Microbiol Today 2010; 37:9.

3. Fukunaga Y, Kurahashi M, Yanagi K, Yokota A, Harayama S. Acanthopleuribacter pedis gen. nov., sp. nov., a marine bacterium isolated from a chiton, and description of Acanthopleuribacteraceae fam. nov., Acanthopleuribacterales ord. nov., Holophagaceae fam. nov., Holophagales ord. nov. and Holophagae classis nov. in the phylum 'Acidobacteria'. Int J Syst Evol Microbiol 2008; 58:2597-2601. PubMed http://dx.doi.org/10.1099/ijs.0.65589-0

4. Bak F, Finster K, Rothfuß F. Formation of dimethylsulfide and methanethiol from the first methyltransferases belong to COG0407, and the second methyltransferases belong to COG1410. $H$. foetida likely uses the same type of process. It has six proteins belonging to COG5012 and 29 proteins belonging to COG0407. The only genome with more members of COG0407 is Mahella australiensis [48] with 33. Some of the COG0407 proteins are found close to corrinoid proteins in the genome sequence. $H$. foetida has two members of COG1410. One is adjacent to the CO dehydrogenase/acetyl-CoA synthase genes and has $61 \%$ identity to the acsE gene of $M$. thermoacetica. It probably transfers methyl groups from tetrahydrofolate to the corrinoid iron-sulfur protein of CO dehydrogenase/acetyl-CoA synthase. The other COG1410 gene is adjacent to a corrinoid protein and may transfer methyl groups from corrinoid proteins to tetrahydrofolate in the methoxyaromatic demethylation pathway.

Livermore National Laboratory under Contract No. DEAC52-07NA27344, and Los Alamos National Laboratory under contract No. DE-AC02-06NA25396, UTBattelle and Oak Ridge National Laboratory under contract DE-AC05-000R22725, as well as German Research Foundation (DFG) INST 599/1-2.

methoxylated aromatic compounds and inorganic sulfide by newly isolated anaerobic bacteria. Arch Microbiol 1992; 157:529-534.

5. Reisch CR, Moran MA, Whitman WB. Bacterial catabolism of dimethylsulfoniopropionate (DMSP). Front Microbiol 2011; 2:172. PubMed

6. Coates JD, Ellis DJ, Gaw CV, Lovley DR. Geothrix fermentans gen. nov., sp. nov., a novel Fe(III)reducing bacterium from a hydrocarboncontaminated aquifer. Int / Syst Bacteriol 1999; 49:1615-1622. PubMed http://dx.doi.org/10.1099/00207713-49-4-1615

7. Altschul SF, Gish W, Miller W, Myers EW, Lipman DJ. Basic local alignment search tool. J Mol Biol 1990; 215:403-410. PubMed

8. Korf I, Yandell M, Bedell J. BLAST, O'Reilly, Sebastopol, 2003.

9. DeSantis TZ, Hugenholtz $P$, Larsen $N$, Rojas $M$, Brodie EL, Keller K, Huber T, Dalevi D, Hu P, Andersen GL. Greengenes, a chimera-checked $16 \mathrm{~S}$ rRNA gene database and workbench compat- 
Anderson et al.

ible with ARB. Appl Environ Microbiol 2006;

72:5069-5072. PubMed

http://dx.doi.org/10.1128/AEM.03006-05

10. Porter MF. An algorithm for suffix stripping. Program: electronic library and information systems 1980; 14:130-137.

11. Lee C, Grasso C, Sharlow MF. Multiple sequence alignment using partial order graphs. Bioinformatics 2002; 18:452-464. PubMed

http://dx.doi.org/10.1093/bioinformatics/18.3.452

12. Castresana J. Selection of conserved blocks from multiple alignments for their use in phylogenetic analysis. Mol Biol Evol 2000; 17:540-552. PubMed

http://dx.doi.org/10.1093/oxfordjournals.molbev.a $\underline{026334}$

13. Stamatakis A, Hoover P, Rougemont J. A rapid bootstrap algorithm for the RAxML web servers. Syst Biol 2008; 57:758-771. PubMed http://dx.doi.org/10.1080/10635150802429642

14. Hess PN, De Moraes Russo CA. An empirical test of the midpoint rooting method. Biol / Linn SoC Lond 2007; 92:669-674.

http://dx.doi.org/10.1111/j.10958312.2007.00864.x

15. Pattengale ND, Alipour M, Bininda-Emonds ORP, Moret BME, Stamatakis A. How many bootstrap replicates are necessary? Lect Notes Comput Sci 2009; 5541:184-200. http://dx.doi.org/10.1007/978-3-642-02008-7_13

16. Swofford DL. PAUP*: Phylogenetic Analysis Using Parsimony (*and Other Methods), Version 4.0 b10. Sinauer Associates, Sunderland, 2002.

17. Pagani I, Liolios K, Jansson J, Chen IM, Smirnova T, Nosrat B, Markowitz VM, Kyrpides NC. The Genomes OnLine Database (GOLD) v.4: status of genomic and metagenomic projects and their associated metadata. Nucleic Acids Res 2012;

40:D571-D579. PubMed http://dx.doi.org/10.1093/nar/gkr1100

18. Ward NL, Challacombe JF, Janssen $\mathrm{PH}$, Henrissat B, Coutinho PM, Wu M, Xie G, Haft DH, Sait H, Badger J. Three genomes from the phylum Acidobacteria provide insight into the lifestyle of these microorganisms in soil. App/ Environ Microbiol 2009; 75:2046-2056. PubMed http://dx.doi.org/10.1128/AEM.02294-08

19. Field D, Garrity G, Gray T, Morrison N, Selengut J, Sterk P, Tatusova T, Thomson N, Allen MJ, Angiuoli SV, et al. The minimum information about a genome sequence (MIGS) specification.
Nat Biotechnol 2008; 26:541-547. PubMed

http://dx.doi.org/10.1038/nbt1360

20. Woese CR, Kandler O, Wheelis ML. Towards a natural system of organisms. Proposal for the domains Archaea and Bacteria. Proc Natl Acad Sci USA 1990; 87:4576-4579. PubMed http://dx.doi.org/10.1073/pnas.87.12.4576

21. Validation List No. 143. Int / Syst Evol Microbiol 2012; 62:1-4.

http://dx.doi.org/10.1099/ijs.0.039487-0

22. Thrash JC, Coates JD. Phylum XVII. Acidobacteria phyl. nov. In: Krieg NR, Staley JT, Brown DR, Hedlund BP, Paster BJ, Ward NL, Ludwig W, Whitman WB (eds), Bergey's Manual of Systematic Bacteriology, Second Edition, Volume 4, Springer, New York, 2010, p. 725.

23. Validation List no. 52. Validation of the publication of new names and new combinations previously effectively published outside the IJSB. Int J Syst Bacteriol 1995; 45:197-198. http://dx.doi.org/10.1099/00207713-45-1-197

24. BAu2A 2010, Classification of bacteria and archaea in risk groups. http://www.baua.de TRBA 466, p. 105.

25. Ashburner M, Ball CA, Blake JA, Botstein D, Butler H, Cherry JM, Davis AP, Dolinski K, Dwight SS, Eppig JT, et al. Gene ontology: tool for the unification of biology. The Gene Ontology Consortium. Nat Genet 2000; 25:25-29. PubMed http://dx.doi.org/10.1038/75556

26. Kreft JU, Schink B. Demethylation and degradation of phenylmethylethers by the sulfidemethylating homoacetogenic bacterium strain TMBS 4. Arch Microbiol 1993; 159:308-315. http://dx.doi.org/10.1007/BF00290912

27. Klenk HP, Göker M. En route to a genome-based classification of Archaea and Bacteria? Syst Appl Microbiol 2010; 33:175-182. PubMed http://dx.doi.org/10.1016/j.syapm.2010.03.003

28. Wu D, Hugenholtz P, Mavromatis K, Pukall R, Dalin E, Ivanova NN, Kunin V, Goodwin L, Wu $M$, Tindall BJ, et al. A phylogeny-driven Genomic Encyclopaedia of Bacteria and Archaea. Nature 2009; 462:1056-1060. PubMed http://dx.doi.org/10.1038/nature08656

29. List of growth media used at DSMZ. http://www.dsmz.de/catalogues/cataloguemicroorganisms/culture-technology/list-of-mediafor-microorganisms.html.

30. Gemeinholzer B, Dröge $\mathrm{G}$, Zetzsche $\mathrm{H}$, Haszprunar G, Klenk HP, Güntsch A, Berendsohn 
WG, Wägele JW. The DNA Bank Network: the start from a German initiative. Biopreserv Biobank $2011 ; 9: 51-55$.

http://dx.doi.org/10.1089/bio.2010.0029

31. The DOE Joint Genome Institute. http://www.jgi.doe.gov

32. Phrap and Phred for Windows. MacOS, Linux, and Unix. http://www.phrap.com

33. Zerbino DR, Birney E. Velvet: algorithms for de novo short read assembly using de Bruijn graphs. Genome Res 2008; 18:821-829. PubMed http://dx.doi.org/10.1101/gr.074492.107

34. Han C, Chain P. Finishing repeat regions automatically with Dupfinisher. In: Proceeding of the 2006 international conference on bioinformatics \& computational biology. Arabnia HR, Valafar $\mathrm{H}$ (eds), CSREA Press. June 26-29, 2006: 141-146.

35. Lapidus A, LaButti K, Foster B, Lowry S, Trong S, Goltsman E. POLISHER: An effective tool for using ultra short reads in microbial genome assembly and finishing. AGBT, Marco Island, FL, 2008.

36. Hyatt D, Chen GL, Locascio PF, Land ML, Larimer FW, Hauser LJ. Prodigal Prokaryotic Dynamic Programming Genefinding Algorithm. BMC Bioinformatics 2010; 11:119. PubMed http://dx.doi.org/10.1186/1471-2105-11-119

37. Pati A, Ivanova N, Mikhailova N, Ovchinikova G, Hooper SD, Lykidis A, Kyrpides NC. GenePRIMP: A Gene Prediction Improvement Pipeline for microbial genomes. Nat Methods 2010; 7:455-457. PubMed http://dx.doi.org/10.1038/nmeth.1457

38. Lowe TM, Eddy SR. tRNAscan-SE: a program for improved detection of transfer RNA genes in genomic sequence. Nucleic Acids Res 1997; 25:955-964. PubMed

39. Lagesen K, Hallin PF, Rødland E, Stærfeldt HH, Rognes T, Ussery DW. RNammer: consistent annotation of rRNA genes in genomic sequences. Nucleic Acids Res 2007; 35:3100-3108. PubMed http://dx.doi.org/10.1093/nar/gkm160

40. Griffiths-Jones S, Bateman A, Marshall M, Khanna A, Eddy SR. Rfam: an RNA family database. Nucleic Acids Res 2003; 31:439-441. PubMed http://dx.doi.org/10.1093/nar/gkg006

41. Krogh A, Larsson B, von Heijne G, Sonnhammer ELL. Predicting transmembrane protein topology with a hidden Markov model: Application to complete genomes. J Mol Biol 2001; 305:567580. PubMed http://dx.doi.org/10.1006/jmbi.2000.4315

42. Bendtsen JD, Nielsen H, von Heijne G, Brunak S. Improved prediction of signal peptides: SignalP 3.0. J Mol Biol 2004; 340:783-795. PubMed http://dx.doi.org/10.1016/j.jmb.2004.05.028

43. Baas D, Rétey J. Cloning, sequencing and heterologous expression of pyrogallol phloroglucinol transhydroxylase from Pelobacter acidigallici. Eur J Biochem 1999; 265:896-901. PubMed http://dx.doi.org/10.1046/j.14321327.1999.00752.x

44. Maclean AM, Haerty W, Golding GB, Finan TM. The LysR-type $\mathrm{PcaQ}$ protein regulates expression of a protocatechuate-inducible ABC-type transport system in Sinorhizobium meliloti. Microbiology 2011; 157:2522-2533. PubMed http://dx.doi.org/10.1099/mic.0.050542-0

45. Kaufmann F, Wohlfarth G, Diekert G. Odemethylase from Acetobacterium dehalogenans - substrate specificity and function of the participating proteins. Eur J Biochem 1998; 253:706-711. PubMed http://dx.doi.org/10.1046/j.14321327.1998.2530706.x

46. Naidu D, Ragsdale SW. Characterization of a three-component vanillate O-demethylase from Moorella thermoacetica. J Bacteriol 2001; 183:3276-3281. PubMed http://dx.doi.org/10.1128/JB.183.11.3276$\underline{3281.2001}$

47. Schilhabel A, Studenik S, Vödisch M, Kreher S, Schlott B, Pierik AJ, Diekert G. The ether-cleaving methyltransferase system of the strict anaerobe Acetobacterium dehalogenans: analysis and expression of the encoding genes. J Bacteriol 2009; 191:588-599. PubMed http://dx.doi.org/10.1128/JB.01104-08

48. Sikorski J, Teshima H, Nolan M, Lucas S, Hammon N, Despande S, Cheng JF, Pitluck S, Liolios K, Pagani I, et al. Complete genome sequence of Mahella australiensis type strain (50-1 BON $^{\top}$ ). Stand Genomic Sci 2011; 4:331-341. PubMed http://dx.doi.org/10.4056/sigs.1864526 